\title{
Linx
}

Revue des linguistes de l'université Paris X Nanterre

$12 \mid 2002$

«Comme la lettre dit la vie »

\section{Signification et Interprétation : l'adjonction de « même »}

Li Yuanhua

\section{(2) OpenEdition}

Journals

Édition électronique

URL : http://journals.openedition.org/linx/1293

DOI : $10.4000 /$ linx.1293

ISSN : 2118-9692

Éditeur

Presses universitaires de Paris Nanterre

\section{Édition imprimée}

Date de publication : 1 octobre 2002

Pagination : 132-139

ISSN : 0246-8743

\section{Référence électronique}

Li Yuanhua, «Signification et Interprétation : I'adjonction de « même » », Linx [En ligne], 12 | 2002, mis en ligne le 10 octobre 2012, consulté le 03 mai 2019. URL : http://journals.openedition.org/linx/1293 ; DOI : $10.4000 / \operatorname{linx} .1293$

Ce document a été généré automatiquement le 3 mai 2019.

Département de Sciences du langage, Université Paris Ouest 


\title{
Signification et Interprétation : l'adjonction de « même »
}

\author{
Li Yuanhua
}

1 L'adverbe même a un rôle grammatical et lexical: grammatical, il fonctionne dans la construction syntactique à titre de mot-outil; lexical, il contribue à la constitution sémantique du discours. Un grand nombre d'analyses montrent déjà quel'adverbe même peut porter, au moins dans certaines situations d'énonciation, sur chaque segment d'un énoncé, plus précisément, qualifier le sujet, le verbe, le complément, l'attribut, le circonstanciel, jusqu'à toute la phrase. Cela signifie que, du point de vue de la théorie de l'information, l'adverbe même comme marqueur de focus est capable de focaliser tout élément à des fins très différentes, et de fusionner sémantiquement avec l'élément auquel il est attaché. Notre approche reposera sur l'hypothèse suivante: l'adjonction est la signification fondamentale et potentielle de l'adverbe même; cette signification serait à la source des autres significations situées dans un «continuum », et appartient donc à la langue; l'appréhension d'une telle signification ne doit ainsi procéder qu'au niveau de la représentation sémantique. A partir de cette compréhension, je me contenterai dans cet article de concentrer mon travail sur l'interprétation d'énoncés contenant même .J'essaierai de montrer dans la première partie que même est un adverbe exprimant l'ajout de gradation, de traiter dans la deuxième la relation entre le sens lexical de même et le contexte, de mettre à jour dans la troisième des principes de la restriction de sélection, et d'aborder enfin la fonction d'implication des énoncés comportant des occurrences de même.

\section{Ajout de gradation exprimé par même}

2 L'adjonction dont il sera question ici est la signification fondamentale et potentielle de l'adverbe même. Ne se logeant pas directement dans le discours, cette signification ne peut être analysée qu'à l'aide du sens du segment affecté par même, constitué dans une interaction avec des éléments du co-texte et du contexte. Par adjonction nous entendons que même confère quelque chose au segment affecté par lui, de sorte que ce segment 
prenne la force argumentative la plus forte des autres qui manifestent la coordination au niveau de structure syntaxique. "Même servant à marquer une gradation et toute gradation est le résultat d'une comparaison » (J. -C. Anscombre 1973 : 63), l'interprétation de l'énoncé contenant une occurrence de même fait ainsi forcément appel aux deux représentations sémantiques plus abstraites et analogues à une structure parallèle en syntaxe: la représentation sémantique de la proposition d'argument fort (i.e.la proposition contenant valeur argumentative plus forte) et la représentation sémantique de la proposition d'argument faible (i. e. la proposition contenant la valeur argumentative plus faible). Ces deux représentations sémantiques constituent un rapport de contraste qui montre que le segment affecté par même a une valeur argumentative plus forte. En d'autres termes, la proposition d'argument fort possède ce qui manque à la proposition d'argument faible du fait de l'ajout signifié par même. Pour bien le voir, examinons un exemple tiré $\mathrm{du}$ « Lexis »:

(1) Il est réservé et même timide.

où deux représentations sémantiques sont possibles :

a) Il est réservé ;

b) Et qui plus est, il est même timide.

3 L'argument " timide » affecté par même et l'argument "réservé » constituent un couple de variables correspondantes l'une à l'autre par rapport aux deux propositions. Ils construisent ainsi une "échelle argumentative", selon le terme d'O. Ducrot, où l'interprétation est régie de la mise sémantiquement en contraste entre eux. Du point de vue de la théorie des Blocs sémantiques proposée par Carel-Ducrot, réservé pourrait être paraphrasé en "croire en lui-même donc ne pas faire ", i. e. assuranceDC NEG-faire, et timide en "ne pas croire en lui-même donc ne pas faire », i. e. NEG-assuranceDC NEG-faire, ils appartiennent donc au même aspect argumentatif $\underline{X D C Y}$ relevant de l'argumentation interne $(\mathrm{AI})^{1}$. On voit par là que l'argument réservé met l'accent sur l'affirmation en faveur du comportement d'une personne, par contre, timide sur la négation. Les deux s'opposent l'un à l'autre, se trouvent ainsi respectivement à un pôle sur "l'échelle argumentative ", et constituent un contraste de valeur argumentatif au sein d'une argumentation. D'où la création d'une gradation sémantiquement hiérarchique dans l'énoncé à l'aide de l'ajout donnée par même.Ce qui permet de faire apparaître la faveur négative que prend l'argument timide.

4 Mais, à la différence d'autres adverbes comme aussi, encore, etc. exprimant l'ajout de quantité dans certaines situations du fait qu'ils traduisent une classe d'anaphores parallèles et que ce qu'ils adjoignent se trouve au même ordre, même ne pourrait pas être compris comme un adverbe exprimant l'addition simple en nombre et en quantité, car il renvoie davantage à l'évolution graduelle dans "l'échelle argumentative» qu'à l'évaluation de quantité. En d'autres termes, comme un "connecteur discursif introduisant l'argument hiérarchiquement prépondérant au sein d'une argumentation " (A. Zribi-Hertz, 1997: 113), même exprime l'ajout de gradation. Considérons les deux énoncés suivants :

(2) Je ne veux pas de gloire! Je ne veux pas de joie! Je ne veux même plus d'espérance! (Georges Bernanos: Sous le soleil de Satan) 
(3) Au bout du mois, il commençait à savoir compter, il lisait presque couramment, et il avait même appris une petite poésie. (Marcel Aymé, Les contes du chat perché)

5 Evidemment, les trois arguments de (1) gloire, joie et espérance, dont la valeur argumentative augmente progressivement le long de « l'échelle argumentative » au fur et à mesure de l'addition des éléments réels, se rapportent à la perception psychologique de mondes possibles. Même a conféré en effet à l'espérance un sens, telle que «l'exigence la plus fondamentale de la vie». Ce qui provoque un changement de la hiérarchie de sorte que l'espérance se place ainsi à un pôle sur « l'échelle argumentative ». Ce changement se manifeste plus clairement dans (2). La poésie est « un art d'évoquer et de suggérer les sensations, les impressions, les émotions par un emploi particulier de la langue » (Lexis), apprendre une poésie est donc un travail mental beaucoup plus complexe et dur par rapport aux deux autres activités. Apprendre une poésie implique alors nécessairement savoir compter et lire, travail plus simple et de moins d'inventivité pour une personne bien instruite. Du point de vue pédagogique, le premier travail intellectuel appartient à un ordre supérieur à celui des autres. Il ressort de là que la proposition d'argument fort « implique » la valeur argumentative que prend la proposition d'argument faible. Soit P le cadre d'une phrase quelconque, $x$ et $y$ deux arguments orientés vers la même conclusion au sein d'une argumentation, on obtiendra à peu près :

$\mathrm{P}(x) \in \mathrm{P}(y)$, si et seulement si $\mathrm{P}(x)$, et même $\mathrm{P}(y)$.

\section{Dénotation et contexte}

6 L'expression du langage se distingue de la mise en œuvre de l'opération logique, la formule $\mathrm{P}(x) \in \mathrm{P}(y)$ que nous considérons ici est seulement de nature d'implication, et entre autres, $\mathrm{P}(y)$ implique nécessairement $\mathrm{P}(x)$ dans un sens strictement logique. Le rapport d'implication entre les arguments corrélatifs ne se manifeste que dans la mesure où les informations de discours prises d'un énoncé sont régies par des conditions particulières.En fait, ces dernières font partie de conditions extralinguistiques construisant l'univers de discours sur lequel s'accordent les participants d'une conversation. L'implication en question se fonde ainsi davantage sur le sens dénotatif des arguments, plus précisément sur la valeur spécifique du sens dénotatif à la manifestation de laquelle contribue même, que sur le sens lexical soi-même des arguments. $\mathrm{P}(y)$ apparait, dans ce sens, comme ayant plus de force argumentative. C'est pour cette raison que l'interprétation d'un énoncé comprenant seulement une proposition d'argument fort doit le plus souvent faire appel à un univers de discours déterminé. D'où une relation logico-sémantique mise de même avec tout autre élément de l'énoncé. Ce qui explique pourquoi même peut apparaître dans des énoncés seuls et ces énoncés peuvent être compris correctement. Il n'est pour voir que de considérer l'exemple suivant :

(4) Même les enfants connaissent cela.

7 J. -C. Anscombre (1973: 50) a fait remarquer que «lorsque l'énoncé comportant une occurrence de même ne s'oppose pas à une phrase antérieure explicite, le segment affecté par même s'oppose toujours à d'autres membres de phrases possibles, mais qui n'ont pas 
été explicités. » Il sera bien possible ici que les adultes, par exemple, soit un des autres membres auxquels l'argument les enfants s'oppose. Normalement, les adultes sont supérieurs aux enfants en développement intellectuel et ont plus de connaissances, plus d'expérience, donc théoriquement, ils doivent connaitre ce que les enfants connaissent et les enfants ne connaissent pas nécessairement ce que les adultes connaissent. Ainsi, même les enfants connaissent cela sous-entend en général que les adultes le connaissent comme de juste. De là, on pourrait déduire que (4) prévoit une représentation sémantique telle :

(4') Les adultes le connaissent naturellement.

Du point de vue de la théorie des Blocs sémantiques, nous pourrons décrire (4) en jeune PT connaître. Ainsi, l'AI (argumentation interne) de (4) est constituée de l'enchaînement LP (linguistiquement paradoxal), (4) est donc LP.L'interprétation prévoit une même représentation sémantique de la proposition d'argument faible : Il est naturel que les adultes le connaissent.

C'est le cas général où l'énoncé traduit une logique des choses, et (4) a alors souvent pour effet de justifier une chose facile à faire. Mais, comme nous l'avons indiqué ci-dessus, l'interprétation d'un énoncé de ce type doit prendre la considération de conditions extralinguistiques, ainsi, (4) n'est pas toujours une expression adéquate dans certaines situations, par exemple au moment où on parle de jouer aux jeux vidéo, bien vulgarisés parmi les enfants, mais que beaucoup d'adultes ne connaissent pas. Donc, l'acceptabilité des énoncés du type de (4) devrait satisfaire les trois conditions nécessaires : a) dans un argument s'incorpore le sens dénotatif qui appartient à une croyance sociale ; b) à l'aide de la valeur spécifique du sens dénotatif évoquée par même, cet argument peut être mis en contraste, selon le contexte, avec un argument correspondant de la proposition d'argument faible, que celui-ci soit implicite ou explicite, afin de déterminer le sens de l'énoncé ; c) ce contraste s'effectue surtout sur le sens dénotatif de ces deux arguments et est limité par l'univers de discours.

On voit par là que le rapport mis de même avec tout élément de l'énoncé n'est pas syntaxique, mais logique, lié à l'expression d'une attitude du locuteur, à la production d'un effet sur l'interlocuteur. Même a ainsi une fonction logico-sémantique, renvoie à des buts et des intentions d'un locuteur. En effet, l'interprétation de l'énoncé contenant une occurrence de même ne consiste pas nécessairement en des enchaînements de discours, mais davantage en un réseau sémantique-cognitif construit par des informations sur le monde et des informations de discours ; tout comme l'indique H. M. Moura (2001: 81), « le sens d'un énoncé est le changement qu'il provoque dans le contexte, et non ses conditions de vérité. Le changement de l'état informationnel correspond à la valeur spécifique du sens dénotatif."

\section{Problèmes de la restriction de sélection}

11 Nous venons de voir comment l'adjonction exprimée par même agit sur la valeur argumentative d'une entité, quel que soit le rôle grammatical de cette entité en catégorie syntaxique : sujet, prédicat, attribut, complément, circonstanciel. On se demande alors dans quelles conditions s'emploie le même en question. En fait, la mise en œuvre 
pertinente de celui-ci dans un énoncé est limitée sous condition de la restriction de sélection qui présente trois principes suivants :

\section{1 Principe de l'échelon}

12 Par principe de l'échelon, nous entendons que l'arrangement à l'égard de $\mathrm{P}(x), \mathrm{P}(y)$ doit s'effectuer selon un certain gradient. Le fait que même exprime l'ajout de gradation permet à $\mathrm{P}(y)$ de « progresser d'un échelon ", c'est-à-dire d'être supérieur à $\mathrm{P}(x)$ en valeur argumentative. Cela veut dire que l'emploi de $\mathrm{P}(y)$ entraîne nécessairement la prise en considération de plus de rapports liés au contexte et à des conditions extralinguistiques. Ainsi donc, l'arrangement correspond forcément au concept d'argument plus fort : « $\mathrm{Si} X$ et $Y$ sont des arguments orientés vers une même conclusion, et si $Y$ est un argument plus fort que $X$ vers cette conclusion, on peut avoir $X$, et même $Y$, mais non $Y$ et même $X »(H . M$. Moura 2001 : 88) De là, il est facile de trouver inacceptable l'arrangement sous forme de $X$, et $Y$, dans des phrases suivantes :

\begin{tabular}{|l|l|l|}
\hline & (5) & Il a l'air heureux et même content. \\
\hline & (6) & Le souffle même lui manqua une minute et il se tut tout d'un coup. \\
\hline
\end{tabular}

13 En évidence, heureux est un argument plus fort que content, qui « est impliqué » par le premier dans une mesure d'intensité du sens lexical : il est sous-entendu qu'un homme heureux, c'est certainement un homme content; par contre, un homme content n'est pas nécessairement heureux. Il faut donc écrire (5) comme :

(5') Il a l'air content et même heureux.

L'explication argumentative de l'inacceptabilité de (6) peut s'opérer par le fait de la contradiction entre les circonstanciels. Minute est une unité de l'heure, destinée à marquer la longueur du temps et tout d'un coup est une locution adverbiale, destinée à signifier l'instantanéité de l'accomplissement d'un acte. En plus, manquer de souffle peut provoquer se taire, c'est-à-dire prévoir le dernier et non inversement. L'ordre de l'arrangement des arguments de (6) a causé donc une contradiction entre les représentations sémantiques des deux propositions, et a transgressé de ce fait l'attente des interlocuteurs lors de la perception d'un événement. De là, nous déduisons que l'énoncé prévu serait plutôt celui ci-dessous :

(6') Il se tut tout d'un coup et le souffle même lui manque une minute.

Ce qui mérite d'être noté ici, c'est que $Y$ est un argument plus fort que $X$ vers une même conclusion ne renvoie pas à la nature connotative du sens lexical des deux arguments, mais à leurs rapports évoqués avec l'univers socioculturel et physico-anthropologique qui structure le mode d'existence du sujet parlant. En d'autres termes, il s'agit là de connaissances sur l'arrière-plan partagées ensemble par les participants l'un et 
l'autre. On voit bien en (7) que le fait que l'argument des plumes jaunes ait de la valeur argumentative la plus forte dépend de l'univers cognitif commun aux participants: la plume jaune est plus difficile à trouver ou bien, elle coûte plus cher, etc. :

(7) J'ai des plumes bleues, des plumes noires et même des jaunes. (Marcel Aymé, Les contes du chat perché)

Nous ferons l'hypothèse à cet effet que s'il y a une série d'arguments $\mathrm{Z}, \mathrm{Y}, \mathrm{X}, \ldots$ orientés vers la même conclusion au sein d'une argumentation, il sera possible d'obtenir une gamme d'arguments dont la valeur argumentative diminue successivement de gauche à droite sur un axe horizontal :

$\mathrm{Z}, \mathrm{Y}, \mathrm{X} \ldots$

Selon la « loi d'abaissement » avancée par O. Ducrot, $\mathrm{Z}$ sera privilégié entre autres par la proposition d'argument fort. Etant donné que la valeur argumentative d'un argument est fonction du choix fait par le locuteur, liée donc à la base socioculturelle et psycocognitive du locuteur, la disposition syntaxique du même argument apparaît comme diverse dans des champs sémantiques différents. Donc, un mot est susceptible de mettre respectivement en contraste avec des mots différents selon le contexte :

Enfants : parents / adultes /maîtres ...

et un groupe de mots d'un même champ sémantique aura aussi probablement deux formes d'arrangement très différentes :

$$
\text { content } \rightarrow \text { satisfait } \rightarrow \text { heureux } \rightarrow \text { ravi ...... (diminution) }
$$

ou : ravi $\rightarrow$ heureux $\rightarrow$ satisfait $\rightarrow$ content ...... (diminution)

\section{2 Principe d'anti-compatibilité}

Le principe d'anti-compatibilité dont il sera question s'apparenterait à la notion d'enchaînement linguistique paradoxal (LP) proposée par Carel et Ducrot. Ce principe régit la construction sémantique en soi de la proposition d'argument fort, exige que des expressions compatibles dans un sens n'apparaissent pas dans l'énoncé contenant une occurrence de même. Ce que nous appelons compatibilité consiste en la rationalité des choses, et non en celle de l'association d'une expression et d'un aspect argumentatif effectuée par la langue, ou par le discours. Nous disons que si mettre en question la justesse d'une hypothèse est une expression appropriée, c'est que l'hypothèse doit être vérifiée par les faits. Ainsi, hypothèse $D C$ douteux est structurellement attaché à hypothèse et appartient au type normatif d'argumentation: $\underline{X D C Y}$. La combinaison de la locution verbale mettre en question avec le nom hypothèse est ainsi harmonieuse et les deux unités linguistiques sont compatibles. Par principe d'anti-compatibilité, nous entendons qu'il n'est pas permis de faire apparaître dans l'énoncé comportant une occurrence de même une combinaison compatible, telle que: Il a mis même en question la justesse de cette hypothèse. Pourtant, le principe d'anti-compatibilité n'exclut pas des expressions présentant l'aspect de «neutres", qui ne sont considérées dans un sens littéral ni comme compatibles, ni comme incompatibles, du fait qu'il n'y a pas une règle objective destinée à régir la combinaison compatible ou non dans une mesure où il s'agit d'un domaine empirique. Par exemple, l'interprétation "raisonnable » ou non de la combinaison du sujet il avec l'attribut heureux dans (5) n'est pas conditionnée par d'autres que de la règle d'évaluation créée par le locuteur lui-même. En fait, (5) est aussi une expression relevant de 
l'incompatibilité, plus précisément de l'incompatibilité implicite qui se traduit dans le contexte, dans la situation d'énonciation, chez le locuteur. Pour cette raison, le principe d'anti-compatibilité n'exige pas que des expressions incompatibles apparaissent nécessairement dans toute proposition d'argument fort, mais exige qu'il ne faut avoir aucune expression compatible. Ainsi, dire que la phrase "Il a mis même en question la justesse de cette hypothèse » est une expression mal exprimée, c'est dire qu'elle a transgressé le principe d'anti-compatibilité.

L'incompatibilité du sens est observable à partir des deux types de combinaison: la combinaison du sujet avec le prédicat et la combinaison se réalisant à l'intérieur du prédicat: essentiellement, celle du verbe avec le complément et celle du verbe avec le circonstanciel.

(8) Tout le monde s'est trompé (et) même les professeurs. (Robert méthodique)

(9) A cette étonnante parole, l'abbé Donissan ne détourna même pas la tête. (Georges Bernanos, Sous le soleil de Satan)

La proposition d'argument fort de (8) peut être décrite comme : même les professeurs se sont trompés, qui évoque un enchaînement de discours comme: Ils sont professeurs, pourtant ils se sont trompés. Censés être érudits, les professeurs sont jugés généralement justes. Ainsi, l'aspect érudit PT se tromper, attaché de façon contextuelle au nom professeur, appartient au type transgressif d'argumentation:X PT Y. En d'autres termes, le sujet professeurs est incompatible avec le verbe se tromper, "même marquant que cette argumentation est transgressive » (M. Carel \& O. Ducrot 1999: 22). La même analyse peut faire apparaître l'incompatibilité de (9), qui est plus remarquable, puisque, intuitivement, il semble normal de détourner la tête à la parole, surtout à cette étonnante parole.

J. -C. Anscombre (1973 : 44) a fait remarquer que «tout énoncé est censé véhiculer une information supplémentaire, apporter quelque chose de plus par rapport aux énonciations le précédant, et donc, d'une certaine façon, tout énoncé est inattendu et étonnant. » Pour un énoncé comportant une occurrence de même, inattendu et étonnant dits incarnent justement une certaine incompatibilité de la combinaison des éléments.

\section{3 Principe de portée}

Le principe de portée régit l'arrangement rationnel à l'égard de même dans la phrase.

J. -C. Anscombre a dit que «deux énoncés ne différant que par la place de même sont susceptibles de recevoir la même interprétation ou des interprétations différentes, toujours selon les conditions d'énonciation. » Il a indiqué ensuite que de tels énoncés sont, « au moins intuitivement, et abstraction faite de la situation d'énonciation, ressentis le plus souvent comme véhiculant des informations différentes ", et qu'il peut très bien se faire que, dans ces deux énoncés, "prononcés dans les mêmes conditions d'énonciation, les effets sémantiques de même soient identiques» ${ }^{2}$. Du point de vue grammatical, le connecteur même comme un marqueur de focus « introduit un constituant focalisé, c'està-dire incarnant dans son énoncé un foyer informatif.» (A.Zribi-Hertz 1990: 113) Il semble ainsi que tout cela doit être dû au fait que même a des noyaux différents. Selon H. Nølke (1993 : 38-57), « le foyer est le résultat de la focalisation », relève d'un fait de la 
parole plutôt que de la langue et, le noyau est désigné dans la signification, fait partie de la structure sémantico-pragmatique de la phrase. «La focalisation n'est aucunement déterminée 'dans' la signification, c'est-à-dire au niveau de la phrase : elle est seulement forcée de se manifester dans le cadre du noyau de l'adverbial, qui, pour sa part, est déterminé déjà 'dans' la signification. » ${ }^{3}$ Il ressort de là que, pour l'interprétation, on doit saisir le noyau de même qui ne peut se trouver qu'à l'intérieur de la portée de cet adverbeci, à savoir du domaine de la phrase sur lequel opère même. Il est nécessaire à cet effet de préciser avant tout l'étendue de la portée de même. Nous inspirant de la règle qui gouverne l'emploi de même, proposée par $\mathrm{H}$. Nølke ${ }^{4}$, il nous semble possible de diviser la portée de même en deux types selon la position syntaxique et ce sur quoi porte même: la portée large, où même prend dans sa portée tout le prédicat même jusqu'à la phrase entière, et la portée restreinte, où même ne porte que sur un certain segment.

La portée large est à considérer comme portée non-marquée. Dans ce cas, le même en question est placé entre l'auxiliaire et le participe/ l'infinitif ou suit immédiatement le verbe du prédicat; l'interlocuteur ne peut alors saisir le foyer informatif évoqué par même qu'à l'aide de l'évocation de l'intonation ou, au moyen de la mise en contraste, selon les conditions d'énonciation, avec un segment antérieur appartenant à la même catégorie syntaxique que le segment sur lequel porte même. Et la portée restreinte est marquée, lorsque l'ordre des mots suffit en général à contribuer à la prise du foyer informatif qui se trouve à l'intérieur du noyau de même, puisque ce noyau est en général toujours le segment qui ou bien précède, ou bien suit immédiatement même, ou vient après en vertu $\mathrm{du}$ « critère de la combinaison des mots voisins ».

Considérons les énoncés dont les parties soulignées sont respectivement la portée de même, et entre autres en italique est le noyau :

\begin{tabular}{|l|l|l|}
\hline$(10)$ & $\begin{array}{l}\text { Pierre a lavé la cuisine, la salle à manger, le salon : il a même lavé la salle de bain. (J. - } \\
\text { C. Anscombre } 1973: 47)\end{array}$ \\
\hline$(11)$ & $\begin{array}{l}\text { Pierre a briqué la cuisine, ciré la salle à manger, repeint le salon : il a mêmelavé la salle } \\
\text { de bain. (ibid.) }\end{array}$ \\
\hline$(12)$ & $\begin{array}{l}\text { Je ne distingue pas en lui, même à présent, la part d'orgueil, de force, de sécheresse ou } \\
\text { de pudeur. (André Gide, L'immoraliste) }\end{array}$ \\
\hline$(13)$ & $\begin{array}{l}\text { Il (Ménalque) semblait fier et ne s'intéressait pas à ma vie. Je fus donc étonné de le voir } \\
\text { à ma première leçon. Son insolence même, qui m'écartait de lui d'abord, me plut, [...] ( } \\
\text { ibid.) }\end{array}$ \\
\hline$(14)$ & $\begin{array}{l}\text { Hé quoi ? La grâce même de Dieu peut-elle être ainsi dupée ? (Georges Bernanos, Sous le } \\
\text { soleil de Satan) }\end{array}$ \\
\hline$(15)$ & $\begin{array}{l}\text { Il regardait à droite et à gauche avec une inquiétude grandissante. Même il se retourna } \\
\text { subitement, et scruta l'ombre, derrière lui. (ibid. ) }\end{array}$ \\
\hline
\end{tabular}

Il est bien clair que les exemples (10), (11) et (15) appartiennent à la portée large de même et que le reste appartient à la portée restreinte de même. Il nous semble plus facile de repérer le noyau de même à la portée restreinte, où il est lié davantage à l'ordre des mots qu'à des situations d'énonciation. En revanche le repérage du noyau de même de (10), de 
(11) et de (15) est beaucoup plus difficile si l'on ne tient pas compte de situations d'énonciation. Si le noyau de même de (10) est différent de celui de (11) bien que même ait la même position syntaxique dans ces deux énoncés, c'est que l'élément antérieur avec lequel est mis en contraste le segment affecté par même a changé. L'information étant donnée par rapport à tout l'énoncé, le verbe laver à la portée large de même de (10) ne peut pas se servir du foyer informatif. Ainsi, le segment la salle de bain est un seul élément susceptible d'être mis en contraste avec le segment correspondant de la proposition antérieure et il est donc plus significatif que l'autre. Mais dans (11), tout le prédicat a fait l'objet du contraste avec celui de la proposition antérieure et entre autres, la valeur argumentative la plus forte est laver la salle de bain. Quant à (15), la relation de contraste étant établie entre deux phrases, la phrase affectée par même est considérée globalement comme noyau de même. La même analyse pourra être faite pour tous les énoncés de ce type.

Il ressort de là que l'interprétation sémantique de l'énoncé comportant des occurrences de même à la portée large dépend davantage de situations d'énonciation (et de l'intonation), alors que dans le cas de la portée restreinte, elle fait appel plutôt à l'ordre des mots. On voit donc de nouveau que, en tout cas, la relation construite de même avec tout élément de la phrase est logico-sémantique, et dépasse de beaucoup celle de la structure grammaticale.

\section{Fonction d'implication de même}

Enfin, nous allons aborder grosso modo deux fonctions de même. A partir de la notion du contexte canonique de l'énonciation, J. -C. Anscombre a bien traité les trois cas de l'emploi de même: le cas idéal du contexte canonique, i.e. le cas où $p$ et $r$ apparaissent explicitement au cours de l'énonciation; le cas où $p$ n'est pas explicite lors de l'acte d'énonciation et le cas où $r$ n'est pas explicite, $p$ étant explicite ou pas ${ }^{5}$. Le changement de la situation de discours est capable en fait de susciter deux fonctions différentes de même: la fonction d'idée (ideational fonction), mise en valeur essentiellement dans le premier cas, et la fonction d'implication (implicational fonction), le plus souvent dans les deux autres. Il en va de même pour la phrase négative.

Comparons les phrases analysées précédemment:

\begin{tabular}{|l|l|l|}
\hline & $(2)$ & $\begin{array}{l}\text { Je ne veux pas de gloire! Je ne veux pas de joie ! Je ne veux même plus d'espérance ! } \\
\text { (Georges Bernanos, Sous le soleil de Satan) }\end{array}$ \\
\hline (8) & Tout le monde s'est trompé (et) même les professeurs. (Robert méthodique) \\
\hline (9) & $\begin{array}{l}\text { A cette étonnante parole, l'abbé Donissan ne détourna même pas la tête. (Georges } \\
\text { Bernanos : Sous le soleil de Satan) }\end{array}$ \\
\hline (4) & Même les enfants connaissent cela. \\
\hline
\end{tabular}

Dans (2) et (8), en faisant confirmer le sens littéral, même met l'accent sur la valeur argumentative du segment affecté par lui en faveur de la justification d'une certaine conclusion. Les énoncés de ce type soulignent donc davantage la vérité de l'information 
véhiculée par q. Et alors dans (9) et (4), c'est en évoquant la relation logique entre $q$ et des mondes possibles selon le contexte que même a mis en relief un certain sous-entendu pris par q. En l'occurrence, il est indifférent que le contenu de l'énoncé soit vrai ou faux, l'important est de soutenir une certaine thèse à l'aide de l'implicature de q. (9) signifierait ainsi : l'abbé Donissan est un homme capable de se bien contenir, et (4) désignerait une chose très facile ou très connue, etc.

Pourquoi $q$ comportant l'occurrence de même a-t-il une fonction d'implication ? Du point de vue des notions de sous-entendu et d'implicature, «il est clair en effet qu'une même phrase peut, quand elle est énoncée, véhiculer à peu près n'importe quel contenu » (O. Ducrot \& J. -M. Schaeffer 1995 : 572). Cela veut dire qu'une même phrase peut bien contenir un certain nombre d'implications potentielles, elles s'opposent l'une à l'autre et une seule peut s'effectuer à chaque fois. De plus, nous l'avons mentionné précédemment, l'interprétation sémantique d'énoncés comportant des occurrences de même se fonde sur le contraste constitué par une série d'arguments entre lesquels apparaît une relation hiérarchique sous l'intervention de l'adjonction signifiée par même. Quand ces arguments sont orientés vers une même conclusion, et que la valeur argumentative de l'argument affecté par même s'est réalisée, la réalisation de la valeur argumentative des autres arguments va de soi, puisque l'argument affecté par même a une valeur argumentative plus forte. Ce qui est l'implicature de $q$. Ainsi donc, dans le premier cas traité par J. C. Anscombre, $q$ comportant une occurrence de même traduit souvent un fait, souligne le sens littéral; l'interprétation repose à cet effet sur la valeur de vérité du contenu sémantique pris par $q$ dans la mesure où la restriction de conditions d'élection a été déterminée; et alors, dans les deux autres cas, $q$ indique souvent l'aspect d'une logique des choses, c'est-à-dire le sens de l'énoncé ; l'interprétation repose alors souvent sur une inférence logique. Car O. Ducrot $(1980: 12)$ a fait remarquer que «la compréhension de l'énoncé implique la découverte de la conclusion précise visée par le locuteur "; l'absence de l'instanciation de $r, p$, surtout de $p$ permet plus de choix des présupposés dans une certaine mesure et entraîne ainsi à faire mettre en rapport $q$ avec un des mondes possibles, en utilisant l'inférence logique pour exclure d'autres mondes possibles. Plus il y aura de présupposés à choisir et de mondes possibles à exclure, plus sera puissante la fonction d'implication de q. Ce qui explique pourquoi des énoncés seuls comportant l'occurrence de même sont le plus souvent implicationnels, qu'ils soient affirmatifs ou négatifs.

Par manque d'espace, nous avons envisagé seulement des situations où l'adverbe même apparait sous forme d'un mot seul dans des énoncés français. Pourtant, cela suffit à montrer que l'adjonction est la signification fondamentale et potentielle de l'adverbe même, et que cette signification contribue non seulement à la construction sémantique, mais à la mise en relief de la fonction pragmatique des phrases.

\section{BIBLIOGRAPHIE}

ANSCOMBRE, Jean-Claude (1973) « Même le roi de France est sage ", Communications 20, 40-82 
BORILLO, Andrée (1962) «La négation et les modifieurs temporels : une fois de plus « encore » », Langue française 62, 37-58.

CAREL, Marion (2001) « Argumentation interne et argumentation externe au lexique : des propriétés différentes », Langages 142, p. 10-21.

CAREL, Marion et DUCROT, Oswald (1999) « Le problème du paradoxe dans une sémantique argumentative ", Langue française 123, 6-26.

CORBLIN, Francis et BEYSSADE, C. (1996) « Présentation », Langages 123, 3-7.

DESCLES, Jean-Pierre, FLAGEUL, Valérie, KEKENBOSCH, Christiane, MEUNIER, Jean-Marc \& RICHARD, Jean-François (1998) « Sémantique cognitive de l'action : 1. contexte théorique », Langages 132, 28-47.

DUCROT, Oswald (1980) « Analyse de textes et linguistique de l'énonciation », Les mots du discours. Paris :Minuit (7-56).

DUCROT, Oswald (1993) Dire et ne pas dire, 3è éd. Paris : Hermann.

DUCROT, Oswald. (2001) «Critères argumentatifs et analyse lexicale », Langages 142, 22-40.

DUCROT, Oswald et CAREL, Marion (1999) «Les propriétés linguistiques du paradoxe : paradoxe et négation ", Langue française 123, 27-40.

DUCROT, Oswald et SCHAEFFER, Jean-Marie (1995) Nouveau dictionnaire encyclopédique des sciences du langage, Paris : Le Seuil.

FUCHS, Catherine (1996) «Struments de temps et struments oncinatifs : L'exemple de encore », Langages $124,73-84$

GARDENT, Claire (1996) « Anaphores parallèles et techniques de résolution », Langages 123, 75-98.

GHIGLIONE, Rodolphe (1998) «Introduction », Langages 132, 3-8.

GREVISSE, Maurice (1993) Le Bon Usage, Grammaire française, Paris : C. F. L.

GROENENDIJK, Jeroen, STOKHOF, Martin, \& VELTMAN, Frank (1996) « Changez le contexte », Langages 123, 8-29.

GROSS, Gaston (1999) «Sémantique lexicale et connecteur », Langages 136, 76-84.

LANDRE, Agnès et FRIEMEL, Edouard (1998) « Opérateurs et enjeux discursifs », Langages 132, 108-123.

M. MOURA, Heronides (2001) «Dénotation et argumentation dans le discours », Langages 142, 77-91

MARQUEZ, Eduardo (1998) «Classification des adjectifs : étude exploratoire sur l'organisation sémantique-pragmatique des adjectifs », Langages 132, 87-107.

MULLER, Claude (1962) «L'association négative », Langue française 62, 59-94.

NØLKE, Hening (1990) «Les adverbiaux contextuels : problèmes de classification », Langue française 88, 12-27.

NøLKE, Hening (1993) Le regard du locuteur, Paris : Kimé.

VICTORRI, Bernard (1999) «Le sens grammatical », Langages 136, 85-105.

ZRIBI-HERTZ, Anne (1990) « Lui-même argument et le concept de « pronom A » », Langages 97, 11-124. 
CUI Xiliang, 1997, 《汉语“连”字句的语用分析》, 新视角汉语语法研究, 北京语言文化大学出版社, 492-508.

JIA Yande, 1999, 《汉语语义学 》, 北京大学出版社.

XU Shenghuan, 1996, «论表添加义的EVEN », 语用问题研究, 河南大学出版社, 158-170.

ZRIBI-HERTZ, Anne (1997).

\section{NOTES}

1. Selon la théorie des Blocs sémantiques, la notion d'argumentation désigne un enchaînement de deux segments de discours, reliés par un connecteur ayant soit la valeur transgressive (représentée par POURTANT, abrégé en PT), soit la valeur normative (représentée par DONC, abrégé en DC). Des enchaînements matériellement différents peuvent réaliser la même relation, soit transgressive soit normative. Ils sont censés alors appartenir à un même aspect, c'est-à-dire un ensemble d'enchaînements. Un aspect peut être évoqué par une entité linguistique. Certains aspects relèvent de l'argumentation interne (AI), d'autres de l'argumentation externe (AE) de l'entité. Dans l'ensemble, l'AI constitue une sorte de paraphrase de l'entité, et l'AE de cette même entité comprend en revanche des aspects où il figure à titre d'antécédent ou de conséquent. Un aspect qui est structurellement attaché à une entité, que ce soit de façon interne ou externe, appartient en effet à la signification linguistique de cette entité, et apparaît donc, en premier plan ou en arrière-plan, dans tous les emplois que l'on fait d'elle. (cf. Ducrot, 2001; Carel-Ducrot, 1999; Carel, 2001).

2. cf. J. -C. Anscombre (1973: 47).

3. cf. H. Nølke (1993 : 38-57).

4. Selon H. Nølke le noyau et la portée de même sont repéré respectivement à partir de la position syntaxique de la manière suivante : A) 1 : Même est prononcé avec l'intonation continuative : Son noyau comprend la combinaison des mots venant à sa suite, jusqu'à la fin du groupe rythmique ; Si même suit immédiatement le verbe (lexical) fini, celui-ci fait partie du noyau. 2 : Même est prononcé avec l'intonation plate: Le syntagme qui le précède immédiatement compose le noyau. B) Si même est placé dans une proposition principale, son champ (ou bien portée) renferme toute la phrase ; 2 : Si même est placé dans une propositionsubordonnée, son champ peut embrasser: soit la proposition subordonnée seulement, soit la subordonnée et la proposition principale, donc la phrase entière. (cf. H. Nølke 1983 : 94, 1993 : 52).

5. Selon J. -C. Anscombre, le contexte canonique de l'énonciation présente la forme suivante :

$r \# p \# q$ (même)

$r$ est la thèse soutenue, $p$ est une série d'arguments avancés à l'appui de $r, q$ est l'énoncé analysé ; la notation $q$ (même) signifie simplement que $q$ est marqué par même, et que même apparaît quelque part à l'intérieur de la chaîne q. Le symbole \#n'est là que pour indiquer que la séparation entre les trois parties est très souvent matérialisée par un procédé intonatoire, ou l'emploi de conjonctions comme ou, et, mais ... etc. (op. cit. p. 55-60) 


\section{AUTEUR}

\section{YUANHUA}

Université du Zhejiang (Hangzhou, Chine) 